Submitted to: Journal of visceral surgery

\title{
Factors of selection and failure of ambulatory incisional hernia repair : a cohort study of 1429 patients
}

Authors: Drissi F ${ }^{1}$, Gillion JF ${ }^{2}$, Cossa JP ${ }^{3}$, Jurczak F ${ }^{4}$, Baayen $C^{5}$; For “Club Hernie”.

\section{Affiliations :}

1. Clinique de Chirurgie Digestive et Endocrinienne (CCDE), Institut des Maladies de I'Appareil Digestif (IMAD), Hôtel Dieu, CHU Nantes, Place Ricordeau, 44093 Nantes cedex 1, France

2. Unité de Chirurgie Viscérale et Digestive, Hôpital Privé d'Antony, 1, Rue Velpeau, 92160 Antony, France

3. Chirurgie Générale et Digestive, CMC Georges Bizet, 23 rue Georges Bizet, 75116 Paris, France

4. Chirurgie générale et digestive, Clinique Mutualiste de l’Estuaire, Cité sanitaire, 11 boulevard Georges Charpak, 44606 Saint Nazaire, France

5. Capionis, 183 avenue de Choisy, 75013 Paris, France

Conflict of interest: FD, JFG, JPC, FJ and CB declare that they have no conflict of interest in relation with this work.

Abstract word count: 255 words

Text word count: 2249 words 


\section{Introduction}

Incisional hernia is a common complication following abdominal surgery with an incidence of $12.8 \%$ after midline incision [1]. It is estimated that about 35000 incisional hernia repairs (IHR) are performed every year in France [2].

In the mid-2000s, the first publications of day-case IHR have emerged [3-8]. Nevertheless, these studies were mainly based on a small number of patients and reported the results of IHR carried out by a laparoscopic approach only. Ambulatory IHR seems to be safe and feasible but no recommendations support this practice so far.

The aim of this study was to assess the ambulatory practice for IHR in France and to identify preoperative factors of selection and situations of non-fulfillment of an ambulatory procedure. 


\section{Method}

\section{Data collection}

A large scale database concerning IHR practice in France was established at the initiative of the "Club Hernie". "Club Hernie" is a group of experienced surgeons, spread across France and particularly familiar with abdominal wall surgery. Within this group, surgeons prospectively collect their data regarding every performed abdominal wall procedure in a dedicated registry [9].

Surgeons of the "Club Hernie" prospectively gathered the data of successive IHR between 08/09/2011 and 22/04/2016. Data concerning patient characteristics were completed preoperatively. ASA grade was determined by the anesthesiologist. Surgical technique employed was left to the discretion of the surgeon. Peroperative items were filled out online in real time after each procedure. Post-operative items were completed along the way of the follow-up.

Data collected in the database were:

- Patient characteristics : age, sex, body mass index (BMI), ASA grade

- Incisional hernia characteristics : preoperative symptoms, site of the incisional hernia, hernia recurrence, hernia width according to the EHS classification (small $<4 \mathrm{~cm}$, medium $4-10 \mathrm{~cm}$, large $>10 \mathrm{~cm}$ ) [10]

- Procedure : surgical technique employed, operating time, ambulatory or inpatient setting, length of stay, complication, grade of the complication according to ClavienDindo [11]

- Causes if ambulatory was not proposed or failed 
The patients were admitted to the hospital in the morning, operated on, monitored and then discharged if they satisfied the conditions of an ambulatory procedure : satisfying vital signs, absence of bleeding or pain, oral intake, deambulation and urination. If not, they were admitted in the surgical department for further surveillance and counted as a failure of the ambulatory setting. Then, each patient was seen by the surgeon one month after the surgery or before if necessary.

One-day surgery was defined as a hospital stay $<12$ hours, with admission and discharge on the same day, according to the current definition of the International Association for Ambulatory Surgery [12]. The ambulatory setting was decided by both the surgeon and the anesthesiologist. The reasons why the patient had not been selected for an ambulatory procedure were reported in the database as well as the causes of failure of a previously selected ambulatory setting.

Complications were divided into medical complications, surgical site collections (SSC) and surgical complications. Early post-operative complications were medical (acute urinary retention, cardiovascular or neurological disorders) or surgical complications likely to delay the discharge of the patient. Infected and uninfected collections (seroma, hematoma) were considered as surgical site collections.

\section{$\underline{\text { Statistical analysis }}$}

Comparisons of qualitative and quantitative data were respectively performed using the chisquared test and Student's T-test. Multivariate regression logistic models were used to identify predictive factors of (i) whether or not an ambulatory procedure was proposed (yes/no) and (ii) whether a proposed ambulatory procedure was successful or not (yes/no). 
To facilitate an easy interpretation of the model coefficients, all numeric variables were centered at their mean value.

Statistical analyses were performed using the statistical software R version 3.3.1 (R: A language and environment for statistical computing. R foundation for Statistical Computing, Vienna, Austria). A statistical significance level of 0.05 was used.

\section{Results}

A total of 1429 patients were operated on for IHR between 08/09/2011 and 22/04/2016. The mean age was $63.3(22-97)$ years. $697(48.8 \%)$ patients were males. The mean BMI was $29.4 \mathrm{~kg} / \mathrm{m}^{2}$ and $481(33.7 \%)$ patients had an ASA grade $\geq 111$. Most of the patients suffered from incisional hernias less than $10 \mathrm{~cm}$ wide. Patients' characteristics are presented in Table 1.

Two hundred and seventy-two (19\%) procedures were performed as an ambulatory surgery (Table 2). Mean length of stay of hospitalized patients was 4.6 days (1-84).

Open IHR were mainly performed (962 patients, 67.3\%). The mean operating time was 54.7 minutes.

A total of 190 (13.3\%) patients experienced at least one complication (Table 3). The most frequent complications were uninfected surgical site collections, broncho-pulmonary complications and small bowel obstructions. There were less complications in the ambulatory group as compared to the inpatients. 
Complications were mainly minor according to the Clavien-Dindo classification [11]. Twentysix (1.8\%) patients experienced serious complications ( $\geq \mathrm{IIIB})$ and $2(0.1 \%)$ of them died. No severe complications or unplanned readmissions were reported in the ambulatory group.

A total of 1124 (78.7\%) patients were not selected preoperatively for an ambulatory procedure. It has been reported that ambulatory was not proposed in about $25 \%$ of the patients because of organizational issues (supporting problems, combined act during the same admission, comprehension or social problem, long distance from hospital, or unavailability of the ambulatory care unit). Medical conditions such as ASA grade $\geq 1 \mathrm{II}$, sleepdisordered breathing or medication relay were other main reasons for not proposing an ambulatory setting for IHR (Table 4).

Age (OR 0.97; $p<0.001$ ), BMI (OR 0.95, $p<0.001)$, ASA grade $\geq$ III (OR 0.23, $p<0.001$ ), hernia width $\geq 4 \mathrm{~cm}$ (OR 0.44, $\mathrm{p}<0.001$ ), recurrent hernia (OR 0.55, $\mathrm{p}=0.01$ ) and laparoscopic IHR $(0.56, p<0.01)$ were identified as factors influencing practitioners for not selecting patients for an ambulatory care (Table 5).

Conversely, 305 patients were selected pre-operatively for an ambulatory procedure. Among these patients initially selected for a one-day procedure, 272 (89.2\%) patients were successfully managed in an ambulatory setting.

In the database, half of the ambulatory failures were caused by medical problems such as pain (10 patients, 33\%), vomiting (6 patients, 18\%) or fainting/headache (2 patients, $6 \%$ ). Social or organizational issues (supporting problem, late check-out from the operating room 
or stress) explained about $15 \%$ of the failures although one-day surgery was initially planned (Table 6).

Although hernia width $\geq 4 \mathrm{~cm}$, laparoscopic incisional hernia repair or occurrence of an early post-operative complication tended to be associated with a failure of ambulatory care, none of these factors reached statistical significance (Table 7).

\section{Discussion}

Little is reported concerning one-day surgery for IHR and the majority of the studies concerned laparoscopic IHR. In this multicenter study, we aimed to assess the practice of ambulatory surgery for IHR in France using a prospective large-scale database. Data concerning 1429 successive IHR have been gathered by senior surgeons, working all around the country, during a period of nearly 5 years. Two hundred and seventy-two (19\%) patients underwent an ambulatory IHR. Only 33 (11\%) of the 305 preoperatively selected patients for ambulatory failed and were finally kept in the hospital for further surveillance. Ambulatory surgery for IHR is safe and feasible but relies on a good preoperative selection of the patients.

Increasing age, $\mathrm{BMI}, \mathrm{ASA}$ grade $\geq \mathrm{III}$, incisional hernia width $\geq 4 \mathrm{~cm}$, recurrent hernia and laparoscopic procedure were identified as pre-operative factors influencing practitioners for not selecting patients for an ambulatory procedure. Ambulatory surgery was sometimes not proposed because of organizational issues which do not endanger the patients. However, none of these factors were identified to be predictive of ambulatory failure. Particularly, IHR of hernias wider than $4 \mathrm{~cm}$ in obese patients appeared to be feasible. 
As a comparison, a previous study of our group highlighted that an ASA grade $\geq 11$, a bilateral hernia repair, an emergency care for incarcerated hernia, a spinal anesthesia or the occurrence of an early postoperative complication were risk factors for ambulatory failure in patients initially selected for one-day surgery [13].

The mean age of the patients was $63.3(22-97)$ years old in the whole cohort. Age alone is not considered as a contra-indication for ambulatory surgery as such. Older patients probably benefit less from outpatient surgery because of higher comorbidities and wider incisional hernias. Qin et al. assessed the incidence of 30-day complications and unplanned readmissions, following outpatient ventral hernia repair, based on the national surgical quality improvement project data files [14]. They highlighted that age and body mass index were predictors of overall complications and surgical complications.

Likewise, ASA grade $\geq 11$ l has been identified as a predictor of unplanned readmissions after outpatient ventral hernia repair (OR 1.46, $p=0.008$ ) [14]. In our experience, only $11 \%$ of patients with an ASA grade $\geq I I I$ underwent an ambulatory IHR and $20 \%$ of the patients were not selected preoperatively for one-day surgery because of such a condition. In France, the health care system does not recommend to consider patients from ASA grade $\geq$ III with unstable disease for ambulatory surgery [15]. Sanjay et al. compared postoperative complications after inguinal hernia repair and did not find significant differences between ASA grades I-II and ASA grades III-IV patients [16]. Inguinal hernia surgery was mainly performed under local anesthesia in this report. As a result, ASA grade III and IV patients should not be strictly contraindicated for one-day surgery.

We also identified the size of the hernia to be paramount in the ambulatory selection. The EHS classification was used in our registry [10]. Hernia width $\geq 4 \mathrm{~cm}$ was identified to be 
predictive of non-selection for an ambulatory care. Indeed, almost $65 \%$ of the patients selected for an ambulatory care suffered from small incisional hernias $(<4 \mathrm{~cm})$ whereas the majority of medium or large incisional hernias were excluded. All the above confirms the external validity of this classification [17]. The hernia size should be definitely considered when selecting the patients for an ambulatory procedure.

One hundred and ninety (13.3\%) patients experienced at least one complication. There were less complications among the patients benefiting from ambulatory surgery as compared to the inpatients. The main complications reported were uninfected superficial subcutaneous collections, broncho-pulmonary complications and small bowel obstructions. Among uninfected superficial subcutaneous collections, the occurrence of a seroma is a common situation, but it usually arises later and thus does not delay the patient outlet. However, the rate of seroma was not higher in the ambulatory group. Two patients suffered from an abdominal compartment syndrome and 2 patients experienced an early recurrence of incisional hernia. These latter rare but serious complications occurred in hospitalized patients. Two patients died of a complication. In their experience concerning 259 consecutive laparoscopic IHR, performed in a short stay surgery department, Lorente-Herce et al. reported a $9.8 \%$ rate of complications [6]. Main complications were intraoperative (intestinal wound, bleeding, need for conversion) or early postoperative ones such as prolonged ileus or hematoma. Fourty $(15.74 \%)$ seroma requiring percutaneous evacuation occurred in this study but were considered as late post-operative complications and were not included in the total complication rate. Three patients experienced serious complications (bile peritonitis, acute peritonitis following an intestinal perforation and intestinal obstruction caused by an inadequate fixation of the mesh) and were reoperated on. They 
only readmitted two patients $(<1 \%)$ due to intra-abdominal hematoma or colo-cutaneous fistula. This data was not gathered at the time of the study in our registry.

One thousand one hundred and fifty-seven (81\%) patients were hospitalized for the management of their incisional hernia. One in four patients were not selected preoperatively for ambulatory IHR because of organizational issues (supporting problems, combined act during the same admission, comprehension or social problem, long distance from hospital or unavailability of the ambulatory care unit). These reasons for not proposing one-day surgery could be reconsidered particularly concerning healthy patients. Selection criteria for one-day surgery should take into account age, body mass index, ASA grade, hernia recurrence and hernia width according to our findings. In their experience, Donati et al. proposed hernia size $<10 \mathrm{~cm}$, reducible hernia sac and negative history for obstructive symptoms as selection criteria for ambulatory IHR performed under local anesthesia [4].

Apart from organizational or social issues, pain and vomiting were the main reasons of ambulatory failure in respectively 30 and $18 \%$ of the patients who failed for initially planned ambulatory setting. Strict anesthetic protocols should be proposed to prevent postoperative pain and vomiting such as early administration of analgesia, loco-regional anesthesia (infiltration of the incisions, TAP-block) and anti-emetic drugs [18].

Unfortunately, some data were missing to better specify the causes of non-selection or failure of ambulatory such as age, hernia width or the need for post-operative drainage. These criteria were reported in the "other causes" or were not available.

To our knowledge, this is the first study reporting one-day surgery for incisional hernia in so many patients operated on regardless of the technique. The data are based on a nationwide registry, rigorously filled-out by experienced surgeons. Three hundred and five patients were 
selected for one-day IHR and 272 achieved ambulatory care. Nevertheless, the global rate of ambulatory surgery for IHR remained modest.

\section{Conclusion}

Based on a nationwide database of 1429 patients operated on for IHR, we assessed the current practice of ambulatory surgery for IHR in France. Only 33 on 305 patients (11\%) failed for a previously planned ambulatory care. Increasing age, body mass index, ASA grade $\geq$ III, hernia width $\geq 4 \mathrm{~cm}$ and laparoscopic IHR were identified as factors influencing practitioners for not proposing an ambulatory care. One-day surgery was sometimes not proposed because of organizational issues which do not endanger the patient. A good preoperative selection, based on objective criteria, is required to achieve ambulatory surgery for IHR. Such a care setting should be systematically proposed for IHR of small incisional hernias $(<4 \mathrm{~cm})$ in young patients with few comorbidities.

\section{References}

[1] Bosanquet DC, Ansell J, Abdelrahman T, et al. Systematic Review and Meta-Regression of Factors Affecting Midline Incisional Hernia Rates: Analysis of 14618 Patients. PLoS ONE 2015;10:e0138745.

[2] e-Pass - Accueil. https://www.epmsi.atih.sante.fr/. Accessed 31 Jan 2017

[3] Abdel-Lah O, García-Moreno FJ, Gutiérrez-Romero JR, Calderón F. Initial experience in the laparoscopic repair of incisional/ventral hernias in an outpatient-short stay surgery unit. Cir Esp 2005; 77:153-8.

[4] Donati M, Gandolfo L, Privitera A, Brancato G, Donati A. Day hospital for incisional hernia repair: selection criteria. Acta Chir Belg 2008;108:198-202. 
[5] Engledow AH, Sengupta N, Akhras F, Tutton M, Warren SJ. Day case laparoscopic incisional hernia repair is feasible, acceptable, and cost effective. Surg Endosc 2007;21:84-6.

[6] Lorente-Herce JM, Marín-Morales J, Jiménez-Vega FJ, et al. Laparoscopic incisional hernia repair in an ambulatory surgery-extended recovery centre: a review of 259 consecutive cases. Hernia 2015;19:487-92.

[7] Moreno-Egea A, Cartagena J, Vicente JP, Carillo A, Aguayo JL. Laparoscopic incisional hernia repair as a day surgery procedure: audit of 127 consecutive cases in a university hospital. Surg Laparosc Endosc Percutan Tech 2008;18:267-71.

[8] Zanghì G, Di Stefano G, Leanza V, Arena M, Di Dio D, Basile F. Incisional hernia in day surgery: our personal experience. G Chir 2012;33:218-20.

[9] Kyle-Leinhase I, Köckerling F, Jørgensen LN, et al. Comparison of hernia registries: the CORE project. Hernia 2018. doi: 10.1007/s10029-017-1724-6. [Epub ahead of print].

[10] Muysoms FE, Miserez M, Berrevoet F, et al. Classification of primary and incisional abdominal wall hernias. Hernia 2009;13:407-14.

[11] Clavien PA, Barkun J, de Oliveira ML, et al. The Clavien-Dindo classification of surgical complications: five-year experience. Ann Surg 2009;250:187-96.

[12] IAAS Initiatives. http://www.iaas-med.com/index.php/iaas-initiatives. Accessed 20 Mar 2018

[13] Drissi F, Jurczak F, Cossa JP, Gillion JF, Baayen C; For "Club Hernie". Outpatient groin hernia repair: assessment of 9330 patients from the French "Club Hernie" database. Hernia 2017. doi: 10.1007/s10029-017-1689-5. [Epub ahead of print].

[14] Qin C, Hackett NJ, Kim JY. Assessing the safety of outpatient ventral hernia repair: a NSQIP analysis of 7666 patients. Hernia 2015;19:919-26.

[15] Haute Autorité de Santé - Chirurgie ambulatoire - socle de connaissances. http://www.has-sante.fr/portail/jcms/c_1242334/fr/chirurgie-ambulatoire-socle-deconnaissances. Accessed 31 Jan 2017

[16] Sanjay $P$, Jones $P$, Woodward A. Inguinal hernia repair: are ASA grades 3 and 4 patients suitable for day case hernia repair? Hernia 2006;10:299-302.

[17] Kroese LF, Kleinrensink G-J, Lange JF, Gillion JF; Hernia-Club. External Validation of the European Hernia Society Classification for Postoperative Complications after Incisional Hernia Repair: A Cohort Study of 2,191 Patients. J Am Coll Surg 2018;226:223-9.

[18] Gao T, Zhang J-J, Xi F-C, et al. Evaluation of Transversus Abdominis Plane (TAP) Block in Hernia Surgery: A Meta-analysis. Clin J Pain 2017;33:369-75. 


\begin{tabular}{l|l}
$\begin{array}{l}\text { Age (years) } \\
\text { Sex }(n, \%)\end{array}$ & $63.3 \pm 14(22-97)$ \\
$\quad-\quad$ Male & $697(48.8)$ \\
$\quad-\quad$ Female & $732(51.2)$ \\
BMI $\left(\mathrm{kg} / \mathrm{m}^{2}\right)$ & $29.4 \pm 6.4(16-75)$ \\
ASA grade $\geq I I I(n, \%)$ & $481(33.7)$ \\
Hernia width $\geq 4 \mathrm{~cm}(\mathrm{n}, \%)$ & $746(52.2)$ \\
Recurrent incisional hernia (n, \%) & $257(18)$
\end{tabular}

Table 1. Patients characteristics. Age and BMI are summarized by their mean, standard deviation and range. Categorical variables are summarized by the number of patients in each category and the corresponding percentages.

\begin{tabular}{c|l}
\hline $\begin{array}{l}\text { Ambulatory procedure }(n, \%) \\
\text { Surgical technique }(n, \%)\end{array}$ & $272(19)$ \\
- Open IHR & $962(67.3)$ \\
- Laparoscopic IHR & $467(32.7)$ \\
Operating time (minutes) & $54.7 \pm 48.8(5-480)$ \\
Complication $(n, \%)$ & $241(16.9)$ \\
- Medical & $71(5)$ \\
- Surgical site collection & $110(7.7)$ \\
- Surgical & $60(4.2)$
\end{tabular}

Table 2. Procedures characteristics. Operating time is summarized by mean, standard deviation and range. Categorical variables are summarized by the number of patients in each category and the corresponding percentages. 


\begin{tabular}{l|ccc} 
Complications & Ambulatory IHR & Inpatient IHR & Total \\
\hline Medical (n, \%) & 0 & $23(2)$ & $23(1.6)$ \\
\hline Broncho-pulmonary & 0 & $13(1.1)$ & $13(0.9)$ \\
Acute urinary retention & 0 & $6(0.5)$ & $6(0.4)$ \\
Cardio-vascular & & & \\
Surgical site collections (,$\%)$ & $7(2.6)$ & $65(5.6)$ & $72(5)$ \\
Uninfected sc col. & 0 & $18(1.5)$ & $18(1.3)$ \\
Uninfected pp col. & $1(0.4)$ & $16(1.4)$ & $17(1.2)$ \\
Infected subcutaneous col. & 0 & $6(0.5)$ & $6(0.4)$ \\
Infected pp col. & & & \\
Surgical (n, \%) & $1(0.4)$ & $12(1)$ & $13(0.9)$ \\
Small bowel obstruction & 0 & $6(0.5)$ & $6(0.4)$ \\
Intraperitoneal & & & \\
abcess/peritonitis & &
\end{tabular}

Table 3. Complications. Only the most common complications are reported. Complications were distinguished by medical complications, surgical site collections or surgical complications. SC, Sub Cutaneous. Col, Collection. PP, Pre-peritoneal.

Ambulatory not proposed

Total no. of patients ( $n, \%)$

Supporting problem

Comprehension/Social problem

Distance from hospital

Combined act

Ambulatory unavailable

$A S A \geq I I I$

Sleep-disordered breathing

Medication relay

Other causes

Multiple causes

\section{4}

118 (10.5)

$45(4)$

36 (3.2)

$68(6)$

$6(0.5)$

\section{$230(20.5)$}

12 (1)

17 (1.5)

330 (29.4)

262 (23.3)

Table 4. Ambulatory not proposed. Specified reasons for not proposing an ambulatory setting are reported. 


\begin{tabular}{l|cccc}
\multicolumn{1}{c}{} & $\begin{array}{c}\text { Selected for } \\
\text { ambulatory }\end{array}$ & $\begin{array}{c}\text { Not selected for } \\
\text { ambulatory }\end{array}$ & OR & $p$ \\
\hline Total no. of patients $(n, \%)$ & $305(21.3)$ & $1124(78.7)$ & - & - \\
Age (years) & 57.4 & 65 & 0.97 & $<0.001$ \\
Male gender $(n, \%)$ & $143(46.9)$ & $554(49.3)$ & 1.01 & 0.85 \\
Body Mass Index $\left(\mathrm{kg} / \mathrm{m}^{2}\right)$ & 27.2 & 30 & 0.95 & $<0.001$ \\
ASA grade $\geq I I I(n, \%)$ & $34(11.1)$ & $447(39.8)$ & 0.23 & $<0.001$ \\
Physical occupation $(n, \%)$ & $36(11.8)$ & $88(7.8)$ & 0.97 & 0.75 \\
Intensive sport practice $(n, \%)$ & $23(7.5)$ & $24(2.1)$ & 2.06 & 0.01 \\
Hernia width $\geq 4 \mathrm{~cm}(n, \%)$ & $108(35.4)$ & $638(56.8)$ & 0.44 & $<0.001$ \\
Recurrent hernia $(n, \%)$ & $32(10.5)$ & $225(20)$ & 0.55 & 0.01 \\
Laparoscopic IHR $(n, \%)$ & $82(26.9)$ & $385(34.2)$ & 0.56 & $<0.001$
\end{tabular}

Table 5. Comparison of patients selected and not selected for ambulatory upon multivariate analysis. Age, BMI and operating time are summarized by their mean, standard deviation and range. Categorical variables are summarized by the number of patients in each category and the corresponding percentages.

Ambulatory failure

\begin{tabular}{l|c}
\hline Total no. of patients ( $\mathrm{n}, \%)$ & 33 \\
Pain & $10(\mathbf{3 0})$ \\
Acute urinary retention & $1(1.7)$ \\
Fainting, headache & $2(6)$ \\
Vomiting & $\mathbf{6 ( 1 8 )}$ \\
& $\mathbf{2 ( 6 )}$ \\
Supporting problem & $1(3)$ \\
Late check-out from operating room & $2(6)$ \\
Stress & \\
& $6(18)$ \\
Other causes & $4(12)$ \\
Multiple causes &
\end{tabular}

Table 6. Causes of ambulatory failures. Specified reasons of ambulatory failure are reported. 


\begin{tabular}{l|cccc}
\multicolumn{1}{c}{} & Outpatient IHR & Ambulatoryfailure & OR & $P$ \\
\hline Total no. of patients & 272 & 33 & - & - \\
Age (years) & 57.5 & 58.3 & 1 & 0.88 \\
Male Sex $(n, \%)$ & $132(48.5)$ & $11(33.3)$ & 2.29 & 0.08 \\
Body Mass Index $\left(\mathrm{kg} / \mathrm{m}^{2}\right)$ & 27.1 & 27.5 & 0.99 & 0.81 \\
ASA grade $\geq$ III $(\mathrm{n}, \%)$ & $30(11)$ & $4(12.1)$ & 1.09 & 0.9 \\
Physical occupation $(n, \%)$ & $34(12.5)$ & $2(6)$ & 1.28 & 0.76 \\
Intensive sport practice (n, \%) & $21(7.7)$ & $2(6)$ & 0.81 & 0.78 \\
Hernia width $\geq 4 \mathrm{~cm}(n, \%)$ & $92(33.8)$ & $16(48.5)$ & 0.50 & 0.10 \\
Recurrent hernia $(n, \%)$ & $27(9.9)$ & $5(15.2)$ & 0.68 & 0.53 \\
Laparoscopic IHR & $70(25.7)$ & $12(36.4)$ & 0.46 & 0.07 \\
Early post-operative & & & & \\
complication $(n, \%)$ & $3(1.1)$ & $2(6)$ & 0.13 & 0.058
\end{tabular}

Table 7. Comparison of outpatient procedures achieved and ambulatory failures upon multivariate analysis. Age and BMI are summarized by their mean. Categorical variables are summarized by the number of patients in each category and the corresponding percentages. 